\title{
IoT Based Home Security System using Atmega328P, ESP01 and ThingSpeak Server
}

\author{
Kaustav Bandyopadhyay ${ }^{1}$, Debasmita Ghosh ${ }^{1}$, Shubhajyoti Ghosh ${ }^{1}$, Soumen Bhowmick ${ }^{1}$, \\ Sumani Mukherjee ${ }^{2}$ and Dr. Rabindranath Ghosh ${ }^{3}$ \\ U.G. Student, ECE Department, St. Thomas' College of Engineering \& Technology, Kolkata, India ${ }^{1}$ \\ Assistant Professor, ECE Department, St. Thomas College of Engineering \& Technology, Kolkata, India ${ }^{2}$ \\ Professor, ECE Department, St. Thomas' College of Engineering \& Technology, Kolkata, India ${ }^{3}$
}

\begin{abstract}
This paper is concerning a home security system based on the Internet of Things (IoT) for providing smart security features as well as automated controls to a house to convert it into a "Smart Home". It uses the inexpensive but powerful microcontroller ESP-01 with Wi-Fi capabilities to control the entire house with the help of a mobile phone having internet access and another microcontroller Atmega328P to control the sensors and actuators in the house. A Thingspeak global web server is used for the storage of data. The hardware uses an infrared sensor along with a Reed switch to detect presence at the front door and monitor the status of the door. Infrared sensors are placed on the inside doors to keep a count of people in a particular room. The model house consists of motorized doors and windows which can be controlled via mobile or automatically from theAtmega328P. The house also consists of relays for switching appliances and a buzzer for alerting the user in a variety of situations. Additionally, there are DHT11 temperature and humidity sensor, MQ2 gas sensor and an LDR to monitor various environmental parameters of the rooms. These sensors give live feeds of temperature, humidity and brightness and alert the user in case of gas leakage. There is a smart monitoring system which switches appliances in any room depending on the number of people present in that room as well as the specific brightness of the room.
\end{abstract}

Keywords: Internet of Things, Thingspeak, Home Security, MIT App Inventor, Atmega328P, ESP01

\section{INTRODUCTION}

Internet of Things (IoT) refers to the infrastructure of connected physical devices through Internet. Recently it is growing at a very rapid pace as a large number of devices are getting associated to the Internet. This technology uses the Internet to integrate the non-intelligent home appliances to work in unison. Home security is a very useful application of IoT and provides essential benefits at an affordable price. This paper presents the application of an Atmega 328P microcontroller to gather inputs from all the sensors and process them into a set of actions which are manifested in the form of security features of the smart home. ESP-01 microcontroller is used to connect this system to a WiFi network in order to keep the user constantly informed about the unusual activities if any. The motorized doors and windows of the house can be controlled via signaling from a smart mobile phone. Any person coming in close proximity to the main door triggers the buzzer to alert the user. Forced entries inside the house usually form the biggest threat to any house. This model will detect these intrusions into the house and send emergency calls to helplines of concerned authorities. The buzzer rings to alert the people inside the house and the doors of the room are automatically shut to keep the inmates safe. There is provision for temperature and humidity measurements along with brightness levels inside the house with the feeds being shown real time on the phone. If there is a fire or gas leakage in the house, the doors and windows will automatically open to let the occupants of the house escape after the buzzer rings. An emergency call will be made to the local fire brigade for help and a notification of the same will be sent to the user in case he is absent from the house at that time. The appliances inside will be also remotely controlled via the phone instead of physical switch boards. Also, we can get live feeds about the number of people in a particular room and this information will be used by the microcontroller to automatically switch off lights and fans in a room with no people inside. Both the microcontrollers used in this project are programmed using the Arduino IDE which supports programming in $\mathrm{C} / \mathrm{C}++$. The application that is present in the user's mobile is developed using MIT App Inventor. This setup uses both the functionality of a local server as well as a global web server. The global server that is being used is a Thingspeak server which communicates with the ESP01 microcontroller using API calls. A local server is simultaneously hosted on the ESP01 that provides LAN connectivity. 


\section{International Advanced Research Journal in Science, Engineering and Technology}

Vol. 6, Issue 5, May 2019

\section{LITERATURE SURVEY}

In paper [1] home electrical system is interfaced to the general-purpose input/output ports of the microcontroller in an embedded system board and their status is passed to the ARM7 with Bluetooth device. They developed an authentication to the system for authorized person to access home appliances. The remote-control function by ARM9 provides help and assistance especially to the disabled and elderly.

Paper [2] uses Zigbee Based Home Automation embedded system. It indicates the elimination of complicated wiring if ZigBee protocol IEEE 802.15.4 wireless standard is used. ZigBee based home automation wireless sensor network is implemented with small initial cost and is useful to control fans, lights and other home appliances.

Md. N. Chowdhury et al used in [3] Raspberry Pi for access control of door and home appliances through Internet. It used an embedded system along with PIR sensor and wireless camera. With the help of this system an evidence of the visitor can be kept as a record if any emergency case or situation occurs.

Paper [4] focuses on building a smart wireless home security system. It sends alerts to the owner by using Internet in case of any trespass and raises an alarm optionally. Besides, the same can also be utilized for home automation by making use of the same set of sensors. The TI-CC3200 Launchpad board has been used. It comes with an embedded micro-controller and an onboard Wi-Fi shield so that all the electrical appliances inside the home can be controlled and managed.

In paper [5] Subhajit Dey et al presented a Web Based Real-Time Home Automation and Security System. It consists of a Microcontroller and an Ethernet module for connecting to a Wi-Fi router. It gives a static IP address to it so that it can use TCP/ IP based communication with other accessing devices connected to the same router.

In paper [6], the authors propose the UID-CoAP architecture. This new architecture is designed to host IoT services on common embedded systems, like usual consumer appliances. They often need to provide a number of sophisticated functions compared to simple sensor nodes. It combines the constrained application protocol (CoAP) with the ubiquitous ID (uID) architecture.

In paper [7], the architecture used is composed of Embedded Internet Systems (EIS). It also uses standard communication protocols. The Service-oriented architecture (SOA) paradigm has been employed. This combination enables the proposed architecture to exchange sensor- and actuator data with an Internet-based cloud as well as a user's local cloud consisting of sensor IoT devices, smart phones and laptops.

In paper [8] a virtual machine (VM) has been developed for wireless sensor nodes. The VM is based on Common Language Infrastructure of .NET Framework so that users can develop a program on the IoT devices using Microsoft Visual Studio and C\# or C++. The users can update the program on the IoT devices through the wireless sensor network.

\section{SYSTEM DESIGN}

The designing methodology of the system has two major portions: software design and hardware design. The hardware is designed by arranging microcontroller, sensors and actuators whereas software design includes programming that is written and uploaded in the microcontroller. The designed system shows microcontroller connected to sensor-modules and actuator-modules for monitoring and controlling household devices. This design section shows how different hardware components are set up. The specifications and information regarding various components are described below. The various functional units used in the system are as follows:

1. Atmega 328P Microcontroller: It is the main processing unit of the model. It is an 8-bit RISC architecture microcontroller with a storage of 32 Kilobytes. It runs at 5 volts at a clock frequency of $16 \mathrm{MHz}$. It is responsible for controlling all the sensors and actuators in the model home. It communicates with the ESP01 via its serial port.

2. ESP01 Microcontroller: It is the first version of the popular ESP8266 series microcontrollers. It is a WiFi SOC that comes built in with the TCP/IP protocol stack and consists of a 32-bit RISC processor running at $80 \mathrm{MHz}$. It is responsible for connecting this model to the internet. All the data from the model reaches the Thingspeak server through it and similarly user commands finally get executed after this microcontroller receives them from the internet and transmits them to the Atmega328P via the serial port.

3. Sensor Modules:

(a) Reed Switch: It is a magnetic switch that switches contacts in the presence of a magnetic field. It is used to detect the opening and closing of the main door.

(b) IR transmitter and receiver: It creates an IR channel when the transmitter and receiver directly face each other. Cutting the IR beam is the indication of an obstruction which is processed by the microcontroller.

(c) DHT11: This module measures the ambient temperature and humidity and feeds the data to the Atmega328P.

(d) MQ2: This is a gas sensor capable of detecting smoke and thereby alerts the microcontroller of a possible fire.

(e) LDR: A light dependent resistor which gives information regarding the brightness of a particular room. 


\section{International Advanced Research Journal in Science, Engineering and Technology}

Vol. 6, Issue 5, May 2019

4. Actuator Modules:

(a) Buzzer: A piezo buzzer module which alerts the user in case of any emergency like fire, burglary etc.

(b) Motors: The doors and windows of the model are motorized and can be automatically or manually controlled.

(c) L293D: This is a motor driver IC that drives the DC motors used in this model home upon receiving signals from the Atmega328P microcontroller.

5. Mobile Application: The mobile application works on a simple algorithm. It reads the data fields written by the ESP01 on the Thingspeak server to get the information of the status of the sensors and actuators of the model house e.g. main door status, temperature etc. When it receives signals from the user, it writes that data to fields on the Thingspeak server that are read by the ESP01. It is designed using the MIT App Inventor.

6. Thingspeak Server: This is a free server hosted on the Thingspeak website. The ESP01 writes sensor and actuator data on specific data fields which are read by the application. Other data fields contain the user response data which is read by the ESP01 to execute user commands.

7. Local Server: This server is hosted on the ESP01 itself and provides the same functionality as the Thingspeak server, with the only difference being that it operates on a local network and not the internet.

\section{IV.SYSTEM ARCHITECTURE AND IMPLEMENTATION}

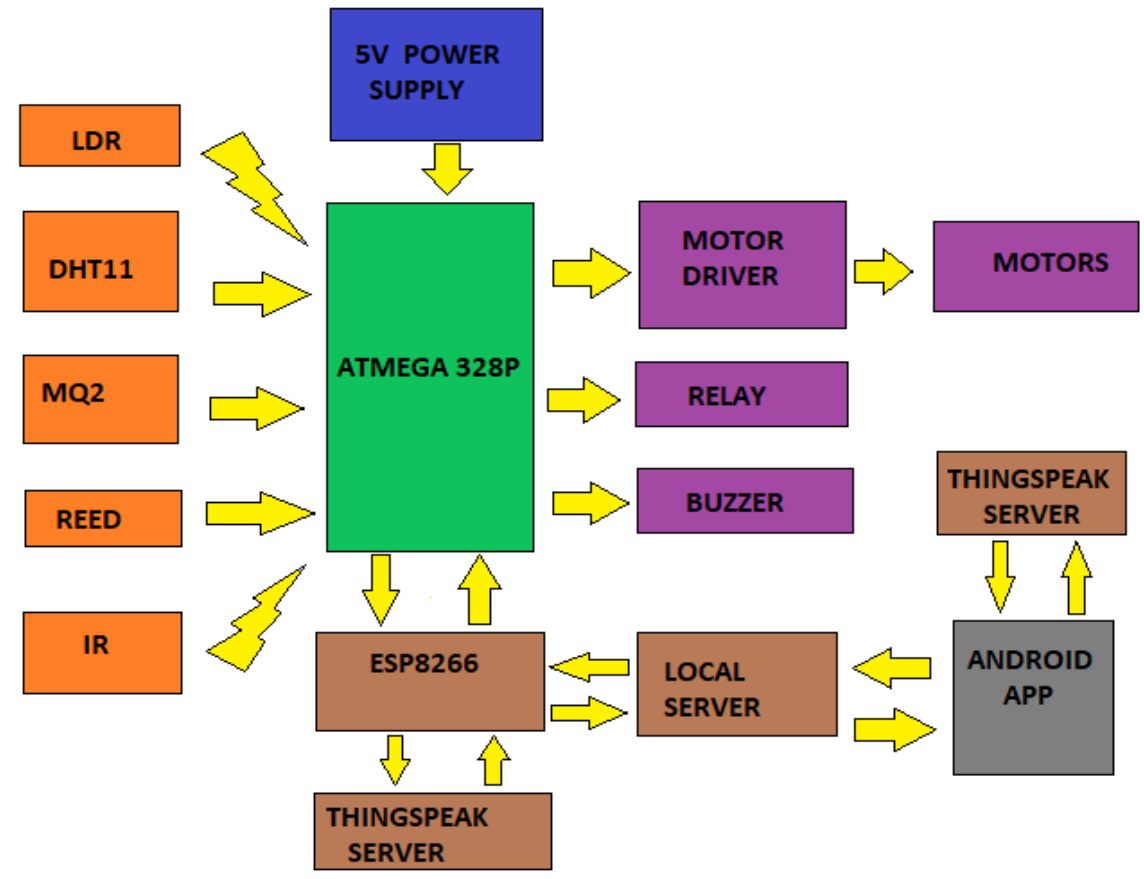

Figure 1: Block Diagram of the System

The block diagram for the model is shown above. The entire home is powered by a 5V DC supply. This supply has been constructed using a step-down transformer, followed by a full bridge rectifier and an LM7805 voltage regulator. The Atmega328P and the ESP01 communicate with each other using Serial Communication protocol. The sensors are interfaced to the digital input pins of the Atmega328P configured in the Arduino IDE. The actuators consisting of motors and buzzer are interfaced to pins configured as output.

The ESP01 connects to the global Thingspeak server and fetches /writes data using HTTP requests. The sensor data are constantly collected and posted to the server every 2 seconds. The Android application communicates with the server every 2 seconds and updates its data fields according to server values and writes data to it. The circuit diagram is shown below:

The entire model functions with a definite algorithm. The system firstly measures the values of all the sensors connected to it. It then checks whether the ESP01 has sent a request to read the data. If true, the Atmega328P sends this data to the ESP01 and in turn receives user commands from it. It then checks whether there is a break-in or a gas leak emergency. If true, then it carries out the procedure to handle an emergency else it carries out the user instructions. The system also keeps a constant check as to whether there is a presence at the front door and rings the buzzer if it detects. Also, it keeps a constant count of the people inside by monitoring the IR sensors and accordingly switches on/off relays. 
International Advanced Research Journal in Science, Engineering and Technology

Vol. 6, Issue 5, May 2019

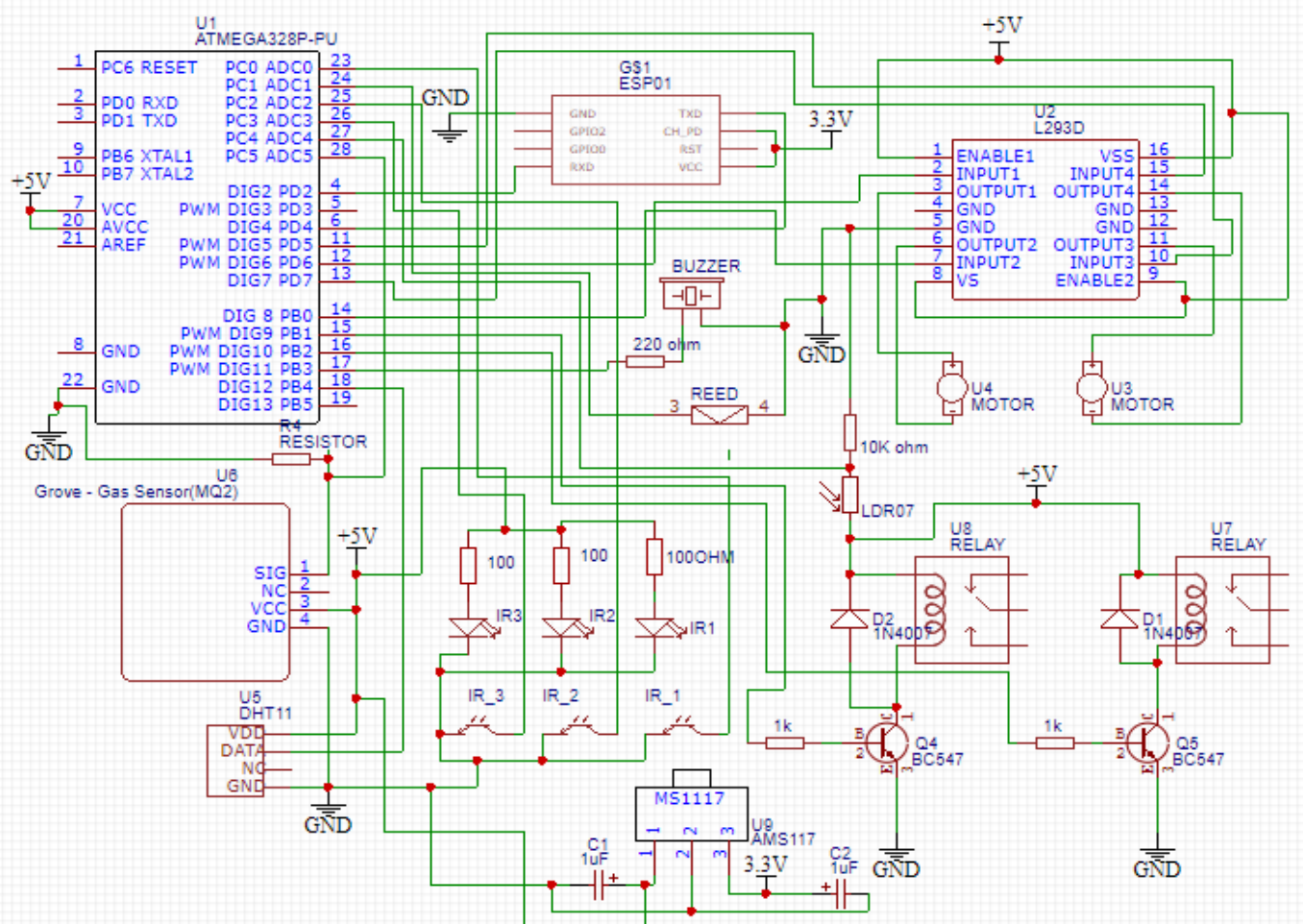

Figure 2: Circuit Diagram of the System

The flowchart is given below:

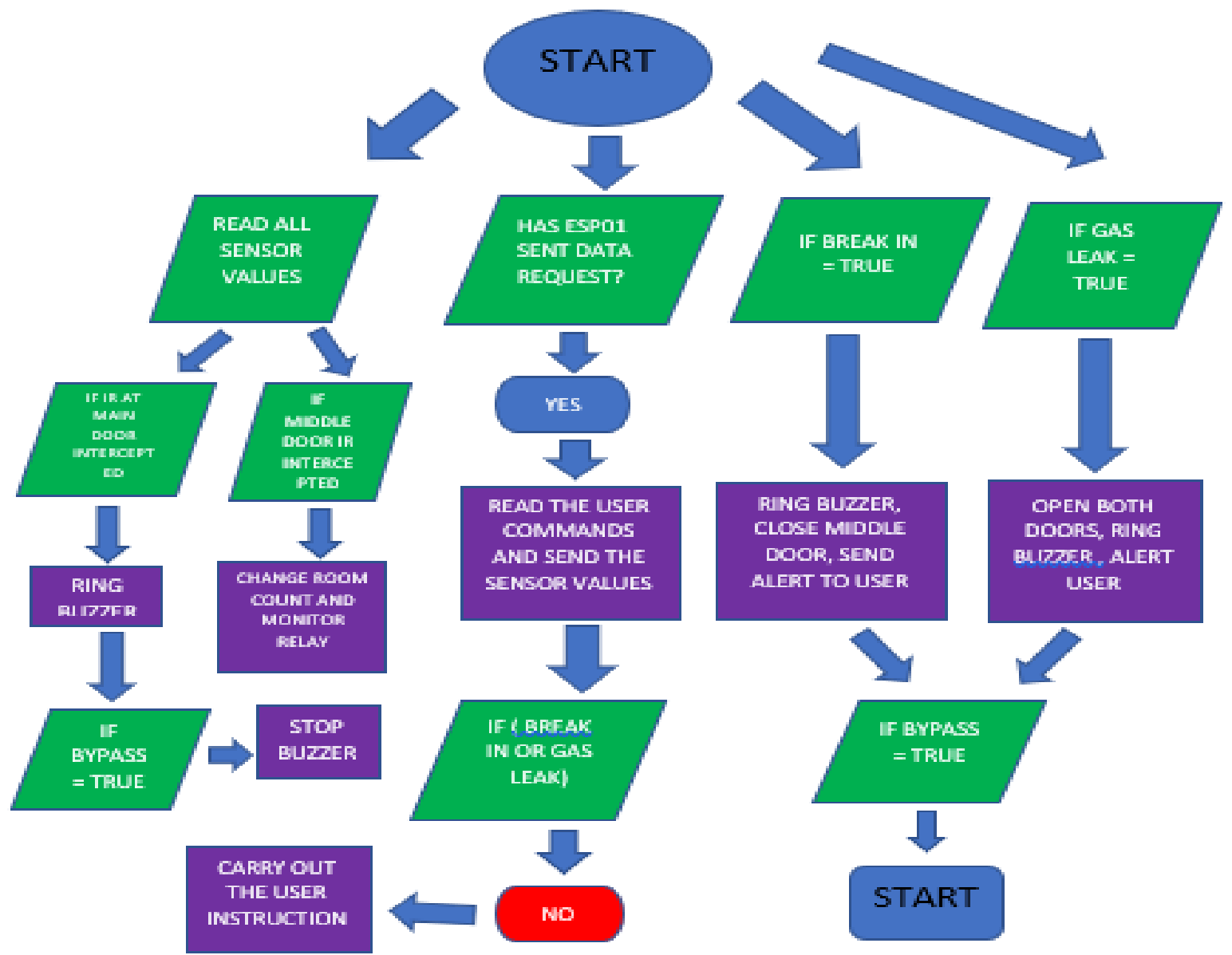

Figure 3: Flowchart of the System 


\section{International Advanced Research Journal in Science, Engineering and Technology}

Vol. 6, Issue 5, May 2019

\section{RESULTS}

The working model is shown below:

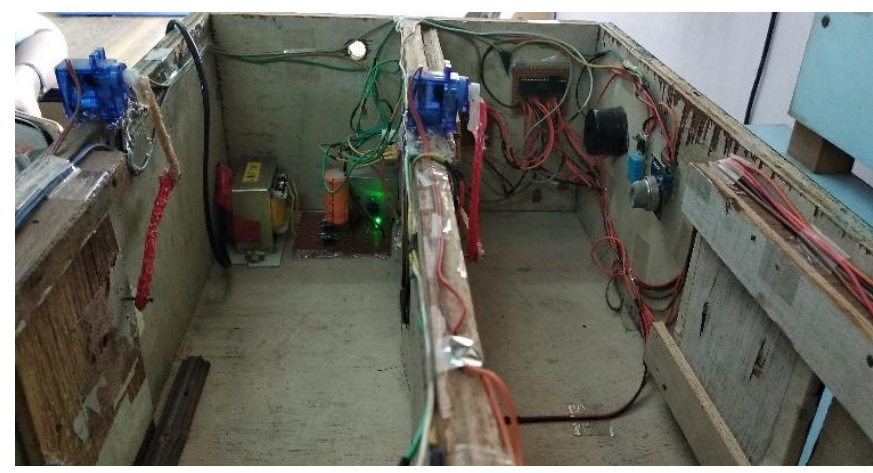

Figure 4: Overview of the Smart Home

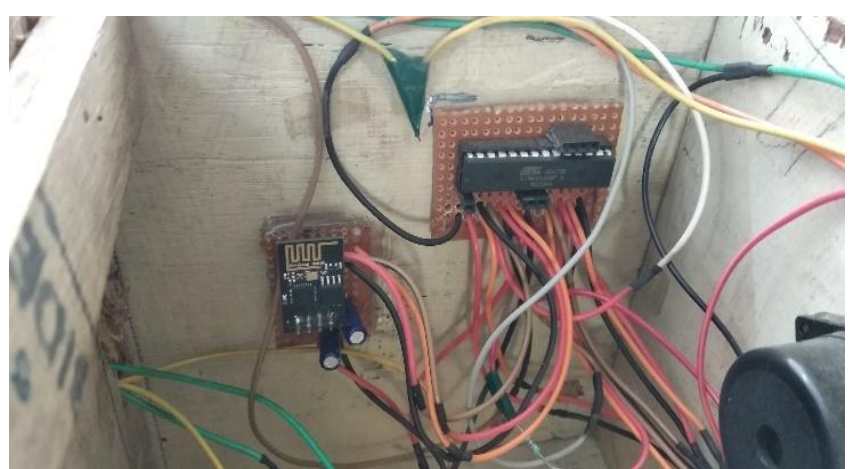

Figure 5: Microcontrollers Atmega328P and ESP01

The application interface when the app is in its normal monitoring state is as below:

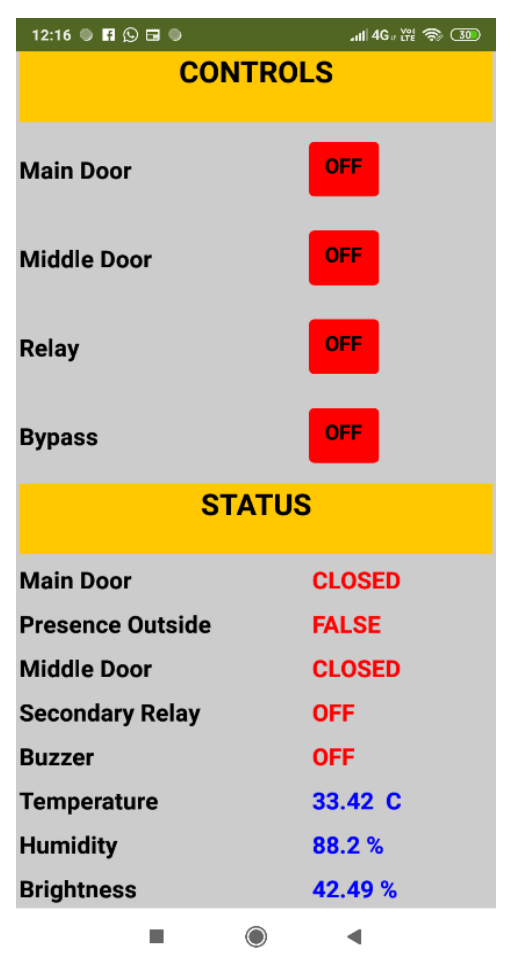

Figure 6: Application layout - 1

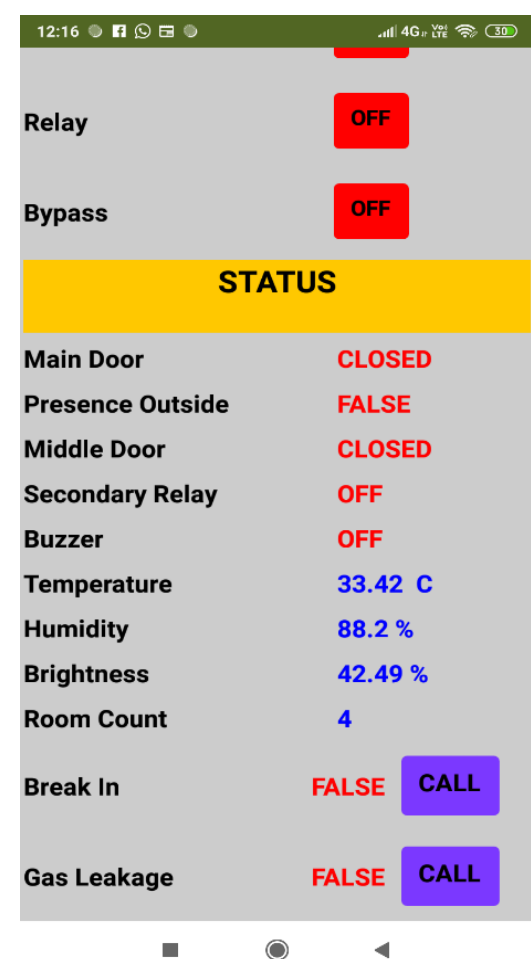

Figure 7: Application Layout - 2 


\section{International Advanced Research Journal in Science, Engineering and Technology}

Vol. 6, Issue 5, May 2019

It can be observed that there are four buttons for controlling the front door, middle door, relay and reset. Apart from that the values of all the sensors are displayed along with the temperature, humidity and brightness feed. There are two call buttons for the break in and gas leak emergencies, in which case on pressing the call button, the application automatically makes a call to an emergency number.

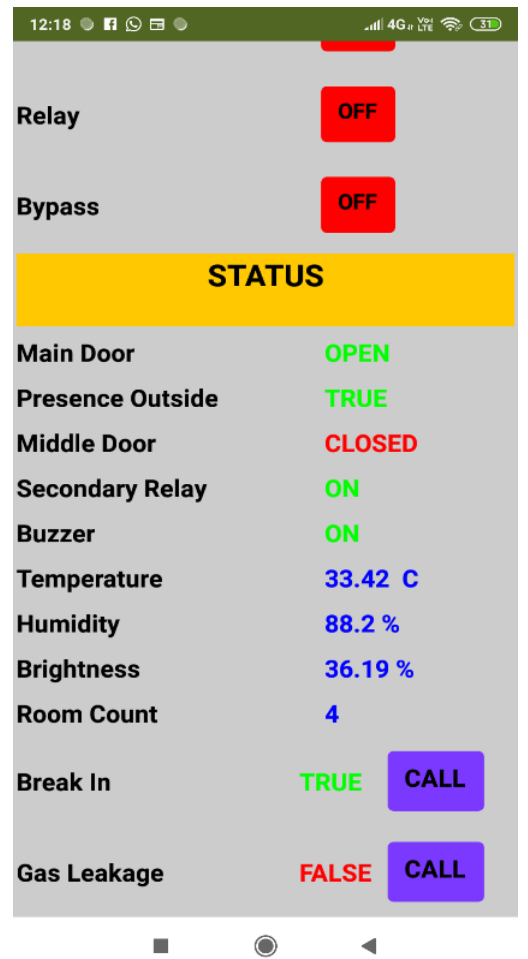

Figure 8: Break-In Condition

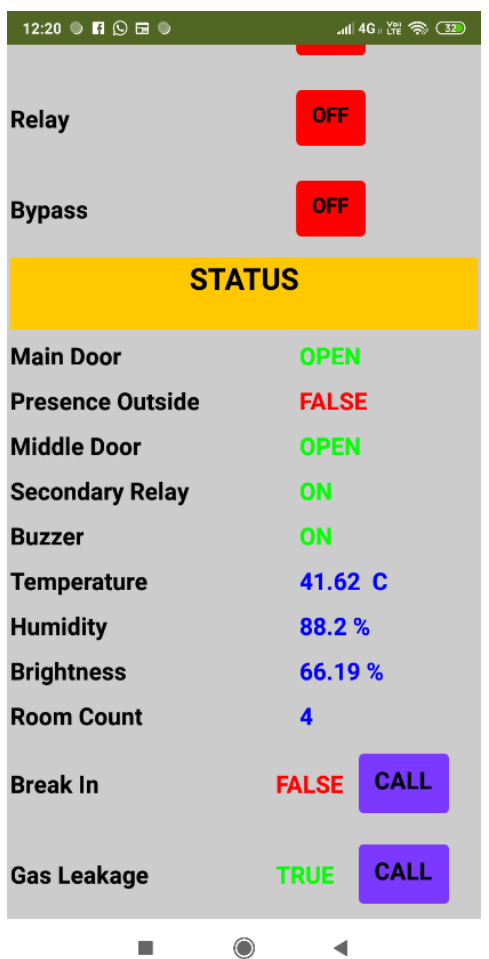

Figure 9: Gas Leakage Condition

The Thingspeak server IoT data representation is shown below
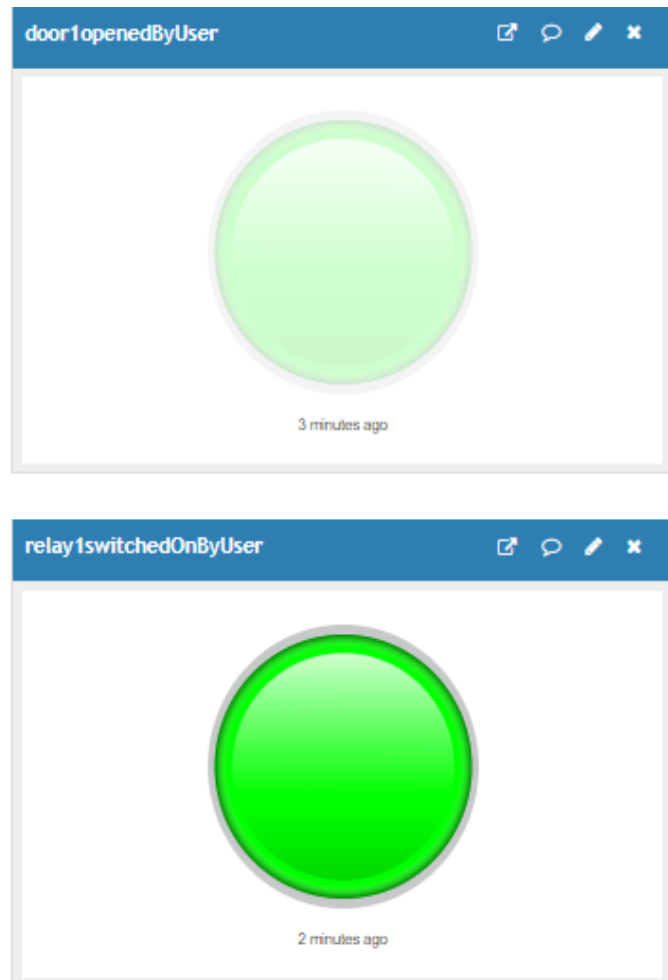

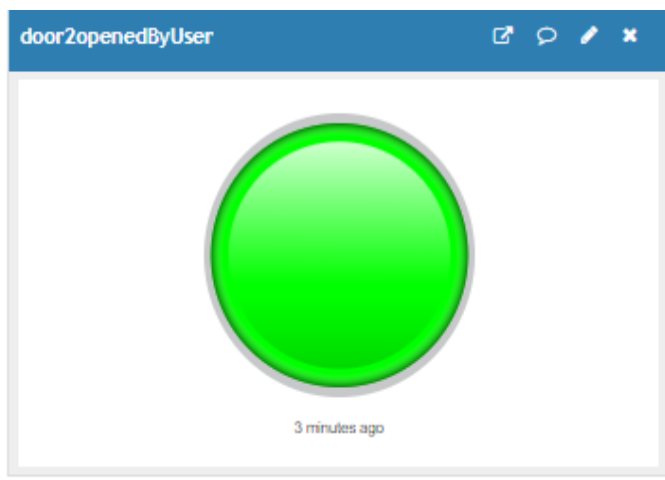




\section{International Advanced Research Journal in Science, Engineering and Technology}

Vol. 6, Issue 5, May 2019
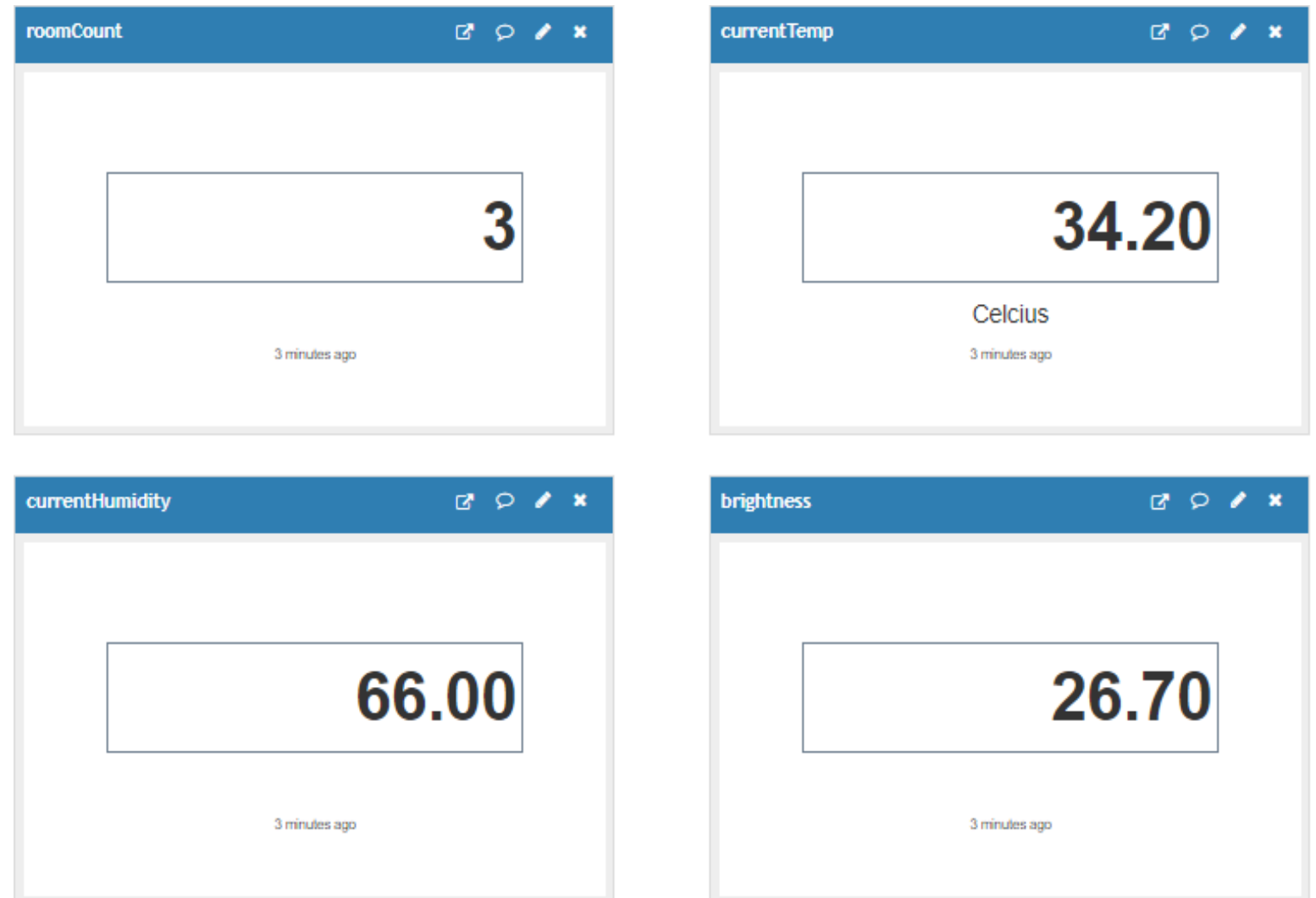

Figure 10: Thingspeak Server Data Fields

\section{CONCLUSION}

This model home provides home security at a much lesser cost compared to the systems available commercially. It has two modes of data transfer - through a local server and through a global server. The Thingspeak data IoT service is used to accomplish the control of the sensors and actuators in the house which resembles the systems that are used commercially. We have implemented IoT that has been implemented along with an option for local communication such as LAN. The smart home is capable of sensing people near a house, providing alerts for gas leakage or a break-in. The option of calling an emergency number from the application in case of these emergencies for faster response is demonstrated. It features temperature, humidity, brightness data and also has a sensing technology whereby it keeps track of people in a room and simultaneously switches on/off appliances based on presence or ambient brightness level. The entire model is powered by a $5 \mathrm{~V}$ power supply which draws an average of $600 \mathrm{~mA} \mathrm{DC}$.

\section{REFERENCES}

[1]. D. Naresh, B.Chakradhar, and S.Krishnaveni, "Bluetooth Based Home Automation and Security System Using ARM9"; International Journal of Engineering Trends and Technology (IJETT); vol 4, issue 9, Sep 2013.

[2]. Jitendra Rana and Sunil P. Pawar, "ZigBee based Home Automation"; SSRN, April 10" 2010.

[3]. Md. N. Chowdhury, Md. S. Nooman and Srijon Sarker, "Access Control of Door and Home Security by Raspberry Pi through Internet"; International Journal of Scientific and Engineering Research; vol 4, issue 11, Nov 2013.

[4]. Ravi Kishore Kodali, Vishal Jain, Suvadeep Bose and Lakshmi Boppana, "IoT Based Smart Security and Home Automation System", National Institute of Technology (NIT), Warangal, April 2016.

[5]. S. Dey, T. Kundu, S. Mukherjee and M. Sarker, "Web Based Real-time Home Automation \& Security System"; IJEETC, vol 4, no 3, Jul. 2015.

[6]. Takeshi Yashiro, Shinsuke Kobayashi, Noboru Koshizuka and Ken Sakamura," An Internet of Things (IoT) Architecture for Embedded Appliances", 2013 IEEE Region 10 Humanitarian Technology Conference, August 2013.

[7]. Pablo Punal Pereira, Jerker Delsing, Asma Raayatinezhad, Jens Eliasson, Rumen Kyusakov, Mia Johansson,” Enabling Cloud-connectivity for Mobile Internet of Things applications", IEEE Seventh International Symposium on Service-Oriented System Engineering, 2013.

[8]. Takayuki Suyama, Yasue Kishino and Futoshi Naya," Abstracting IoT devices using virtual machine for wireless sensor nodes", Internet of Things (WF-IoT), 2014 IEEE World Forum, Seoul, 2014. 